\title{
Climate-associated changes in mercury sources in the Arctic fjord sediments
}

Ju Hyeon Lee ${ }^{1}$, Sae Yun Kwon ${ }^{1,2 *}$, Hoin Lee ${ }^{1}$, Seung-Il Nam ${ }^{3}$, Jung-Hyun Kim ${ }^{3}$, Young Ji $\mathrm{Joo}^{3,4}$, Kwangchul Jang ${ }^{3}$, Haryun Kim ${ }^{5}$, Runsheng Yin ${ }^{6}$

1 Division of Environmental Science and Engineering, Pohang University of Science and Technology, 77 Cheongam-Ro, Nam-Gu, Pohang 37673, South Korea 2 Institute for Convergence Research and Education in Advanced Technology, Yonsei University, 85 Songdogwahak-Ro, Yeonsu-Gu, Incheon 21983, South Korea 3 Division of Glacial Environment Research, Korea Polar Research Institute, 26 Songdomirae-Ro, Yeonsu-Gu, Incheon 21990, South Korea 4 Now at Department of Earth and Environmental Sciences, Pukyong National University, 45 Yongso-Ro, Nam-Gu, Busan 48513, South Korea

5 East Sea Research Institute, Korea Institute of Ocean Science and Technology, 48 Haeyangwahakgil, Jukbyeon, Uljin 36315, South Korea 6 State Key Laboratory of Ore Deposit Geochemistry, Institute of Geochemistry, Chinese Academy of Sciences, 99 West Lincheng Road, Guiyang, Guizhou 550081, China

\footnotetext{
* Corresponding author: Sae Yun Kwon

Phone: +82-54-279-2290

Fax: +82-54-279-8299

Email: saeyunk@postech.ac.kr
} 
This supporting information contains 7 pages and 5 tables.

Table of Contents:

Table S1. Lithology and geological properties of Dicksonfjorden and Woodfjorden bedrocks.

Table S2. Data sources of the biogeochemical measurements (TOC, $\delta^{13} \mathrm{C}$ and $\varepsilon \mathrm{Nd}$ ) of Dicksonfjorden and Woodfjorden sediment cores.

Table S3. Mercury isotope ratios of the standard reference materials measured in this study.

Table S4. THg concentration, TOC, THg/TOC, and Hg isotope ratios of JM05-046-GC (JM05) sediment core.

Table S5. THg concentration, TOC, THg/TOC, and Hg isotope ratios of HH12-964-GC (HH12) sediment core. 
Table S1. Lithology and geological properties of Dicksonfjorden and Woodfjorden bedrocks.

\begin{tabular}{|c|c|c|c|c|c|c|c|}
\hline $\begin{array}{c}\text { Location } \\
\text { (Coordinate) }\end{array}$ & Sediment core & $\begin{array}{l}\text { Length } \\
\text { (cm) }\end{array}$ & $\begin{array}{c}\text { Age } \\
(\text { ka BP })\end{array}$ & Age & Bedrock lithology & Core lithology & $\begin{array}{c}\text { Mean } \\
\text { sedimentation } \\
\text { rates }(\mathrm{cm} / \mathrm{ka})\end{array}$ \\
\hline \multirow[t]{4}{*}{$\begin{array}{c}\text { Dicksonfjorden } \\
\left(78^{\circ} 40.50^{\prime} \mathrm{N}\right. \\
\left.15^{\circ} 18.89^{\prime} \mathrm{E}\right)\end{array}$} & JM05-046-GC & 299 & 10.8 & $\begin{array}{c}\text { Carboniferous, } \\
\text { Permian and } \\
\text { Devonian }\end{array}$ & $\begin{array}{l}\text { Red sandstone, } \\
\text { black mudstone } \\
\text { and calcareous } \\
\text { shale }\end{array}$ & & 28 \\
\hline & & $0-92$ & $0-2.8$ & & & Mud with infrequent clast & \\
\hline & & $92-192$ & $2.8-6.6$ & & & $\begin{array}{l}\text { Mud with five intervals of } \\
\text { sand and clast larger than } \\
2 \mathrm{~mm}(>5 \mathrm{~cm} \text {-thick) }\end{array}$ & \\
\hline & & $192-299$ & $6.6-10.8$ & & & Mud with scattered clast & \\
\hline Woodfjorden & HH12-964-GC & 334 & 13.3 & Devonian & Red sandstone & Silt-sized sediment & 25 \\
\hline $\begin{array}{c}\left(79^{\circ} 39.04^{\prime} \mathrm{N}\right. \\
\left.13^{\circ} 45.3^{\prime} \mathrm{E}\right)\end{array}$ & & 110 & 5.6 & & & $\begin{array}{l}\text { Occurrence of coarse } \\
\text { sediment layers }\end{array}$ & \\
\hline
\end{tabular}


Table S2. Data sources of the biogeochemical measurements (TOC, $\delta^{13} \mathrm{C}$ and $\varepsilon N d$ ) of Dicksonfjorden and Woodfjorden sediment cores.

\begin{tabular}{ccccc}
\hline Location & Sediment core & TOC & $\boldsymbol{\delta}^{\mathbf{1 3}}$ & ENd \\
\hline \multirow{2}{*}{ Dicksonfjorden } & JM05-046-GC & O & O & HH16-964-GC \\
& Reference & Joo et al. (2019) & Joo et al. (2019) & Jang et al. (2020) \\
Woodfjorden & HH12-964-GC & $\mathrm{O}^{\mathrm{a}}$ & $\mathrm{X}$ & O \\
& Reference & This study & N/A & Jang et al. (2020)
\end{tabular}

${ }^{a}$ Total organic carbon (TOC) concentrations were measured at the State Key Laboratory of Ore Deposit Geochemistry, Institute of Geochemistry, Chinese Academy of Sciences, China following the Walkley-Black procedure (Nelson and Sommers, 1996). All samples were measured in duplicates and the standard deviations of sample duplicates were within $5 \%$. 
Table S3. Mercury isotope ratios of the standard reference materials measured in this study.

\begin{tabular}{|c|c|c|c|c|c|c|c|c|c|c|c|c|c|c|c|c|c|c|c|}
\hline Standard & $\mathbf{n}$ & $\delta^{204} \mathrm{Hg}$ & 2SD & $\delta^{202} \mathrm{Hg}$ & 2SD & $\delta^{201} \mathrm{Hg}$ & 2SD & $\delta^{200} \mathrm{Hg}$ & 2SD & $\delta^{199} \mathrm{Hg}$ & 2SD & $\Delta^{204} \mathbf{H g}$ & 2SD & $\Delta^{201} \mathrm{Hg}$ & 2SD & $\Delta^{200} \mathrm{Hg}$ & 2SD & $\Delta^{199} \mathrm{Hg}$ & 2SD \\
\hline RM 8610 & 42 & -0.80 & 0.14 & -0.53 & 0.08 & -0.44 & 0.09 & -0.26 & 0.05 & -0.15 & 0.05 & -0.01 & 0.09 & -0.04 & 0.07 & 0.00 & 0.04 & -0.02 & 0.04 \\
\hline TORT-3 & 4 & 0.06 & 0.19 & 0.10 & 0.10 & 0.57 & 0.15 & 0.11 & 0.05 & 0.64 & 0.12 & -0.09 & 0.04 & 0.50 & 0.08 & 0.06 & 0.02 & 0.62 & 0.10 \\
\hline MESS-4 & 2 & -3.09 & 0.03 & -2.07 & 0.03 & -1.57 & 0.03 & -1.02 & 0.01 & -0.48 & 0.05 & 0.00 & 0.02 & -0.01 & 0.05 & 0.01 & 0.03 & 0.04 & 0.06 \\
\hline
\end{tabular}


Table S4. THg concentration, TOC, THg/TOC, and Hg isotope ratios of JM05-046-GC (JM05) sediment core.

\begin{tabular}{|c|c|c|c|c|c|c|c|c|c|c|c|c|c|}
\hline $\begin{array}{c}\text { Depth } \\
(\mathrm{cm})\end{array}$ & $\begin{array}{l}\text { Age } \\
\text { (ya) }\end{array}$ & $\begin{array}{c}\delta^{204} \mathbf{H g} \\
(\%)\end{array}$ & $\begin{array}{c}\delta^{202} \mathrm{Hg} \\
(\%)\end{array}$ & $\begin{array}{c}\delta^{201} \mathrm{Hg} \\
(\%)\end{array}$ & $\begin{array}{c}\delta^{200} \mathrm{Hg} \\
(\%)\end{array}$ & $\begin{array}{c}\delta^{199} \mathrm{Hg} \\
(\%) \\
\end{array}$ & $\begin{array}{c}\Delta^{204} \mathrm{Hg} \\
(\%)\end{array}$ & $\begin{array}{c}\Delta^{201} \mathbf{H g} \\
(\% o)\end{array}$ & $\begin{array}{c}\Delta^{200} \mathrm{Hg} \\
(\%)\end{array}$ & $\begin{array}{c}\Delta^{199} \mathrm{Hg} \\
(\%)\end{array}$ & $\begin{array}{c}\text { THg } \\
(\mathrm{ng} / \mathrm{g})\end{array}$ & $\begin{array}{c}\text { TOC } \\
(\text { wt \%) }\end{array}$ & $\begin{array}{l}\text { THg/ } \\
\text { TOC }\end{array}$ \\
\hline 4 & 113 & -1.57 & -1.04 & -0.89 & -0.51 & -0.32 & -0.02 & -0.11 & 0.01 & -0.06 & 25.65 & 0.36 & 70.47 \\
\hline 10 & 282 & -1.41 & -0.94 & -0.84 & -0.46 & -0.35 & 0.00 & -0.13 & 0.01 & -0.11 & 21.82 & 0.37 & 59.07 \\
\hline 14 & 394 & -1.24 & -0.80 & -0.67 & -0.39 & -0.31 & -0.05 & -0.07 & 0.02 & -0.11 & 18.98 & 0.34 & 55.15 \\
\hline 22 & 619 & -1.55 & -1.08 & -0.91 & -0.53 & -0.35 & 0.06 & -0.10 & 0.01 & -0.08 & 19.01 & 0.37 & 50.96 \\
\hline 36 & 1014 & -1.73 & -1.12 & -0.90 & -0.52 & -0.34 & -0.07 & -0.07 & 0.05 & -0.07 & 20.04 & 0.38 & 52.27 \\
\hline 40 & 1126 & -1.70 & -1.12 & -0.95 & -0.56 & -0.35 & -0.03 & -0.11 & 0.00 & -0.06 & 20.55 & 0.42 & 49.26 \\
\hline 42 & 1182 & -1.37 & -0.88 & -0.69 & -0.44 & -0.30 & -0.05 & -0.03 & 0.00 & -0.07 & 18.19 & 0.36 & 50.90 \\
\hline 50 & 1408 & -1.56 & -1.05 & -0.89 & -0.53 & -0.36 & 0.01 & -0.10 & 0.01 & -0.09 & 17.88 & 0.34 & 52.04 \\
\hline 56 & 1577 & -1.95 & -1.26 & -1.01 & -0.61 & -0.43 & -0.06 & -0.06 & 0.03 & -0.11 & 24.18 & 0.44 & 54.36 \\
\hline 58 & 1633 & -1.84 & -1.24 & -1.13 & -0.65 & -0.42 & 0.01 & -0.20 & -0.03 & -0.11 & 19.27 & 0.45 & 42.76 \\
\hline 60 & 1689 & -1.57 & -1.03 & -0.92 & -0.52 & -0.39 & -0.03 & -0.14 & 0.00 & -0.13 & 21.22 & 0.41 & 51.28 \\
\hline 72 & 2027 & -1.96 & -1.31 & -1.16 & -0.66 & -0.45 & 0.00 & -0.17 & 0.00 & -0.12 & 19.64 & 0.44 & 44.91 \\
\hline 86 & 2565 & -1.75 & -1.19 & -1.01 & -0.59 & -0.38 & 0.02 & -0.12 & 0.00 & -0.08 & 22.20 & 0.41 & 54.02 \\
\hline 90 & 2719 & -1.79 & -1.19 & -1.02 & -0.59 & -0.40 & -0.01 & -0.12 & 0.01 & -0.10 & 16.36 & 0.37 & 44.76 \\
\hline 92 & 2796 & -1.95 & -1.31 & -1.08 & -0.65 & -0.38 & 0.02 & -0.09 & 0.01 & -0.05 & 20.47 & 0.40 & 51.51 \\
\hline 98 & 3027 & -1.77 & -1.18 & -1.02 & -0.59 & -0.36 & -0.01 & -0.13 & 0.00 & -0.06 & 18.14 & 0.42 & 43.03 \\
\hline 128 & 4181 & -1.93 & -1.24 & -1.00 & -0.61 & -0.36 & -0.07 & -0.07 & 0.02 & -0.05 & 22.66 & 0.49 & 46.67 \\
\hline 132 & 4334 & -2.53 & -1.72 & -1.38 & -0.88 & -0.47 & 0.04 & -0.09 & -0.01 & -0.04 & 30.28 & 0.48 & 62.94 \\
\hline 136 & 4488 & -2.52 & -1.68 & -1.33 & -0.84 & -0.44 & -0.01 & -0.07 & 0.00 & -0.02 & 26.23 & 0.47 & 55.31 \\
\hline 140 & 4642 & -1.98 & -1.30 & -1.08 & -0.64 & -0.40 & -0.04 & -0.10 & 0.01 & -0.07 & 20.41 & 0.50 & 40.71 \\
\hline 156 & 5257 & -1.93 & -1.26 & -1.04 & -0.63 & -0.39 & -0.05 & -0.09 & 0.00 & -0.07 & 18.36 & 0.54 & 33.92 \\
\hline 160 & 5411 & -1.92 & -1.28 & -1.08 & -0.62 & -0.38 & -0.02 & -0.12 & 0.02 & -0.06 & 17.75 & 0.53 & 33.40 \\
\hline 178 & 6103 & -1.78 & -1.19 & -1.05 & -0.60 & -0.38 & 0.00 & -0.15 & 0.00 & -0.07 & 18.29 & 0.51 & 36.20 \\
\hline 190 & 6565 & -1.95 & -1.33 & -1.14 & -0.67 & -0.43 & 0.04 & -0.14 & -0.01 & -0.10 & 20.47 & 0.51 & 40.26 \\
\hline 194 & 6719 & -1.68 & -1.14 & -0.98 & -0.56 & -0.35 & 0.01 & -0.12 & 0.01 & -0.07 & 18.03 & 0.53 & 33.95 \\
\hline 204 & 7103 & -1.78 & -1.20 & -1.00 & -0.61 & -0.36 & 0.01 & -0.10 & 0.00 & -0.05 & 22.19 & 0.51 & 43.57 \\
\hline 212 & 7411 & -1.83 & -1.24 & -1.08 & -0.62 & -0.39 & 0.01 & -0.15 & 0.01 & -0.08 & 20.84 & 0.48 & 42.99 \\
\hline 220 & 7719 & -1.83 & -1.21 & -1.00 & -0.60 & -0.34 & -0.02 & -0.09 & 0.01 & -0.04 & 22.08 & 0.56 & 39.43 \\
\hline 222 & 7795 & -1.52 & -1.04 & -0.91 & -0.52 & -0.31 & 0.03 & -0.12 & 0.01 & -0.05 & 20.20 & 0.52 & 38.61 \\
\hline 226 & 7949 & -1.84 & -1.24 & -1.01 & -0.61 & -0.36 & 0.00 & -0.08 & 0.01 & -0.05 & 18.61 & 0.52 & 35.78 \\
\hline 238 & 8465 & -1.91 & -1.28 & -1.04 & -0.65 & -0.39 & 0.01 & -0.08 & 0.00 & -0.06 & 19.24 & 0.48 & 40.22 \\
\hline 244 & 8749 & -1.82 & -1.20 & -0.97 & -0.61 & -0.37 & -0.02 & -0.06 & 0.00 & -0.06 & 17.99 & 0.44 & 41.18 \\
\hline 262 & 9510 & -1.83 & -1.23 & -1.01 & -0.62 & -0.38 & 0.01 & -0.09 & 0.00 & -0.07 & 15.46 & 0.53 & 28.99 \\
\hline
\end{tabular}


Table S5. THg concentration, TOC, THg/TOC, and Hg isotope ratios of HH12-964-GC (HH12) sediment core.

\begin{tabular}{|c|c|c|c|c|c|c|c|c|c|c|c|c|c|}
\hline $\begin{array}{c}\text { Depth } \\
\text { (cm) }\end{array}$ & $\begin{array}{l}\text { Age } \\
\text { (ya) }\end{array}$ & $\begin{array}{c}\delta^{204} \mathbf{H g} \\
(\% 0)\end{array}$ & $\begin{array}{c}\delta^{202} \mathrm{Hg} \\
(\% 0)\end{array}$ & $\begin{array}{c}\delta^{201} \mathbf{H g} \\
(\% o)\end{array}$ & $\begin{array}{c}\delta^{200} \mathrm{Hg} \\
(\% \text { o })\end{array}$ & $\begin{array}{c}\delta^{199} \mathbf{H g} \\
(\%)\end{array}$ & $\begin{array}{c}\Delta^{204} \mathbf{H g} \\
(\% \%)\end{array}$ & $\begin{array}{c}\Delta_{(\% o)}^{201} \mathbf{H g} \\
(\%)\end{array}$ & $\begin{array}{c}\Delta_{(\% o)}^{200} \mathbf{H g} \\
(\%)\end{array}$ & $\begin{array}{c}\Delta_{(\% o)}^{199} \mathbf{H g} \\
(\%)\end{array}$ & $\begin{array}{c}\text { THg } \\
\text { (ng/g) }\end{array}$ & $\begin{array}{c}\text { TOC } \\
(\text { wt \%) }\end{array}$ & $\begin{array}{l}\text { THg/ } \\
\text { TOC }\end{array}$ \\
\hline 0 & 0 & -0.97 & -0.70 & -0.63 & -0.37 & -0.27 & 0.07 & -0.11 & -0.02 & -0.09 & 39.30 & 0.85 & 46.24 \\
\hline 5 & 0 & -0.95 & -0.65 & -0.60 & -0.33 & -0.27 & 0.02 & -0.11 & 0.00 & -0.11 & 39.90 & 0.90 & 44.34 \\
\hline 15 & 354 & -1.61 & -1.11 & -1.03 & -0.59 & -0.40 & 0.05 & -0.20 & -0.03 & -0.13 & 19.67 & 0.99 & 19.87 \\
\hline 25 & 1062 & -1.45 & -0.96 & -0.85 & -0.49 & -0.36 & -0.01 & -0.13 & -0.01 & -0.12 & 17.63 & 0.95 & 18.56 \\
\hline 30 & 1416 & -1.10 & -0.76 & -0.58 & -0.38 & -0.30 & 0.03 & -0.01 & 0.01 & -0.11 & 17.06 & 1.08 & 15.80 \\
\hline 35 & 1770 & -1.00 & -0.61 & -0.48 & -0.30 & -0.28 & -0.08 & -0.02 & 0.00 & -0.13 & 16.92 & 1.12 & 15.11 \\
\hline 45 & 2081 & -1.71 & -1.16 & -1.05 & -0.58 & -0.47 & 0.02 & -0.17 & 0.01 & -0.18 & 16.74 & 1.15 & 14.56 \\
\hline 80 & 3822 & -1.87 & -1.26 & -1.10 & -0.63 & -0.46 & 0.00 & -0.15 & 0.01 & -0.14 & 14.79 & 0.91 & 16.25 \\
\hline 85 & 4113 & -1.57 & -1.06 & -0.95 & -0.54 & -0.41 & 0.01 & -0.16 & 0.00 & -0.14 & 12.15 & 0.55 & 22.08 \\
\hline 95 & 4695 & -1.73 & -1.16 & -1.02 & -0.58 & -0.42 & 0.00 & -0.15 & 0.01 & -0.13 & 11.57 & 0.56 & 20.65 \\
\hline 100 & 4986 & -1.96 & -1.32 & -1.08 & -0.65 & -0.44 & 0.00 & -0.09 & 0.01 & -0.10 & 12.08 & 0.61 & 19.81 \\
\hline 105 & 5277 & -1.84 & -1.24 & -1.07 & -0.62 & -0.44 & 0.01 & -0.14 & 0.00 & -0.12 & 12.67 & 0.60 & 21.11 \\
\hline 110 & 5568 & -1.48 & -1.00 & -0.88 & -0.48 & -0.44 & 0.01 & -0.13 & 0.02 & -0.19 & 14.57 & 0.70 & 20.82 \\
\hline 120 & 6150 & -1.66 & -1.09 & -1.06 & -0.53 & -0.45 & -0.04 & -0.24 & 0.02 & -0.18 & 14.31 & 0.56 & 25.69 \\
\hline 135 & 7023 & -1.45 & -1.01 & -0.91 & -0.51 & -0.40 & 0.06 & -0.15 & 0.00 & -0.15 & 13.37 & 0.53 & 25.23 \\
\hline 145 & 7605 & -1.65 & -1.12 & -0.99 & -0.57 & -0.44 & 0.03 & -0.15 & -0.01 & -0.16 & 12.64 & 0.50 & 25.28 \\
\hline 150 & 7896 & -1.52 & -1.03 & -0.94 & -0.53 & -0.41 & 0.02 & -0.17 & -0.01 & -0.15 & 14.91 & 0.35 & 42.59 \\
\hline 165 & 8768 & -1.41 & -0.99 & -0.96 & -0.51 & -0.46 & 0.06 & -0.22 & -0.01 & -0.21 & 10.33 & 0.42 & 24.59 \\
\hline 170 & 9059 & -1.00 & -0.67 & -0.68 & -0.34 & -0.35 & 0.00 & -0.17 & -0.01 & -0.18 & 11.46 & 0.53 & 21.81 \\
\hline 185 & 9932 & -1.31 & -0.91 & -0.86 & -0.47 & -0.38 & 0.04 & -0.18 & -0.02 & -0.16 & 10.62 & 0.31 & 34.27 \\
\hline
\end{tabular}

